\title{
Percepciones de los estudiantes de Psicología de la Fundación Universitaria del Área Andina, modalidad virtual, frente a un curso de alfabetización digital basado en ambientes virtuales de aprendizaje*
}

Perceptions of psychology students from the Foundación Universitaria del Área Andina, virtual form, regarding a digital literacy course based on virtual learning environments

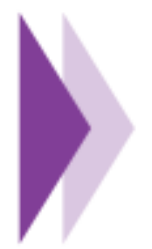

Adelaida Bedoya $^{1}$; Jose Estevez ${ }^{2}$

1 Fundación Universitaria del Área Andina

2 Fundación Universitaria del Área Andina

Correo electrónico: dita.96@gmail.com jestevez@areandina.edu.co 


\section{Resumen}

La presente investigación analiza las percepciones de los estudiantes de Psicología de la Fundación Universitaria del Área Andina en modalidad virtual frente a un curso de alfabetización digital basado en ambientes virtuales de aprendizaje (AVA). El proceder metodológico se fundamenta en un enfoque cuantitativo descriptivo de tipo transversal. La muestra es de 131 estudiantes entre los 16 y los 54 años pertenecientes a los estratos socioeconómicos del 1 al 4. Para la recolección de la información se diseñó y validó un cuestionario que mide la percepción del aprendizaje de contenidos digitales. Los resultados muestran que el nivel de escolaridad previo al inicio del curso es una variable que puede afectar las percepciones de los estudiantes frente a este.

Palabras clave: alfabetización digital, percepción de aprendizaje, competencias digitales, AVA, TIC.

Esta investigación surgió de una necesidad sentida e identificada en estudiantes de primer semestre de Psicología, modalidad virtual, de la Fundación Universitaria del Área Andina. Si bien es cierto que los estudiantes están familiarizados con diferentes entornos digitales, para muchos es la primera experiencia de participación en un entorno virtual de aprendizaje (AVA). También se identificaron otros factores que afectaban el rendimiento, tales como la edad de los estudiantes, el tiempo transcurrido entre el bachillerato y el inicio del pregrado, y la región. Por lo tanto, se diseñó un curso de alfabetización digital orientado a fortalecer los conocimientos en el manejo de las TIC y de diferentes herramientas para el aprendizaje.

Se han tenido en cuenta los avances implementados por el Ministerio de Educación Nacional (MEN) (2006), que ha trabajado en la formulación de estándares básicos de competencias en tecnología e informática como parte de los requerimientos de la Ley 115 de 1994 (Ley General de Educación), en la cual se establece el área de tecnología e informática como obligatoria y fundamental para la educación básica y media, y demarca la importancia de abordar la educación en tecnología como elemento constitutivo de la educación básica y media de niños, niñas y jóvenes. Si bien se han dado muchos avances y alcances a través de las políticas del Gobierno Nacional y el MEN, también se ha identificado que muchos de los estudiantes que ingresan al programa, en diferentes regiones, especialmente las más alejadas de las grandes urbes, presentan debilidades en los conocimientos y en las competencias tecnológicas e informáticas. De ahí la necesidad de diseñar e implementar el curso de adaptación al manejo de las TIC para los estudiantes del programa virtual de Psicología.

El objetivo de este estudio es analizar las percepciones de los estudiantes del programa de Psicología, modalidad virtual, frente al aprendizaje en el curso de alfabetización digital. Para conceptualizar las competencias digitales se tomó la taxonomía de Magro et ál. (2014) para diseñar 25 proposiciones con escalamiento tipo Likert que miden las percepciones de los estudiantes al finalizar el curso. La metodología de esta investigación es cuantitativa, de tipo descriptivo, con diseño transversal y muestreo intencional.

En cuanto a la estructura del presente documento, en un primer apartado se definen los referentes teóricos más importantes, acto seguido se exponen la metodología y los análisis estadísticos del instrumento, después se muestran los resultados y, finalmente, las conclusiones del estudio.

\section{Abstract}

This research analyzes the perceptions of psychology students from the Fundación Universitaria del Area Andina, virtual form, regarding a digital literacy course based on virtual learning environments (VLE). The methodological procedure is based on a cross\#sectional quantitative-descriptive approach. The sample consisted of 131 students between 16 and 54 years old, who belong to socioeconomic strata 1 to 4 . To collect information, a questionnaire was designed and validated that measures the perception of learning digital content. The results show that the level of schooling prior to the start of the course is a variable that can affect students' perceptions of the course.

Keywords: digital literacy, learning perception, digital competencies, VLE, ICT.

\section{El curso de alfabetización digital}

Este curso surgió de la necesidad de desarrollar conocimientos y habilidades tecnológicas en los estudiantes de Psicología modalidad virtual de la Fundación Universitaria del Área Andina, quienes inician su proceso de formación profesional. Este se diseñó en la plataforma Moodle, espacio que tiene la universidad para los procesos de formación complementaria de docentes y administrativos.

Las plataformas como Moodle también suelen ser llamadas LMS, por sus siglas en inglés: Learning Management System, el cual es un sistema de gestión de aprendizaje alojado en un servidor desde donde se proporcionan servicios y herramientas de carácter pedagógico o formativo (Lozano, 2008, citado por Ardila, Ruiz y Castro, 2015). Para Miranda (2004) los sistemas de gestión de aprendizaje o LMS poseen un impacto significativo en el proceso de formación, ya que son herramientas que se pueden emplear para administrar las tareas de los estudiantes. En estas, la comunicación es personalizada y efectiva, además permiten obtener información a través de reportes, evaluaciones y actividades académicas, que son complementadas con lecciones, ejercicios, foros, chats, juegos $\mathrm{y}$ videoconferencias.

\section{Estructura de los contenidos del ambiente de aprendizaje}

En el módulo 1 se aborda el cambio de paradigma frente a la educación virtual, el rol, las expectativas y las competencias que debe tener un estudiante de virtualidad, al igual que el papel del docente. En el módulo 2 se realiza un abordaje en el uso de herramientas y software como: Mindomo, Cmaptools, Canva, Timeline, entre otros, para enseñar a los estudiantes cómo se realizan líneas de tiempo, mapas mentales, mapas conceptuales, infografías, pósteres, entre otros. En el módulo 3 se desarrollan las normas APA a través de las facilidades que presenta Word para su implementación y uso en los trabajos escritos, además de enseñar el uso de bases de datos gratuitas y buscadores de información académica. Por último, el módulo 4 se basa en el uso de herramientas del correo de Gmail, donde los estudiantes aprenden a usar: Hangouts, Meet, YouTube (canal privado) y Drive para el almacenamiento; adicional a esto, se otorgan herramientas como creación de Wikis, creación de formularios en Drive, análisis de datos, revisión de gráficas arrojadas por el cuestionario y compartir enlaces para el diligenciamiento de estos. 


\section{Competencia digital}

Teniendo en cuenta la orientación y los contenidos del curso, es importante definir el término competencia digital y las subdimensiones que lo componen. Para Poriet y Martínez (2014) las competencias tecnológicas son conocimientos, habilidades, destrezas y actitudes que se requieren para usarlas adecuadamente. Así mismo, una competencia tecnológica se define como tener los conocimientos y habilidades para entender, hacer uso y tomar decisiones acerca de la tecnología (Castellanos, Jiménez y Domínguez, 2009).
Las competencias tecnológicas, según Palomo (2001), permiten desarrollar una tecnología competitiva y poseer conocimientos sobre: 1) la razón de ser de la tecnología; 2) los efectos culturales, sociales, económicos, políticos y ambientales de la tecnología; 3) el diseño e ingeniería de procesos, productos y servicios; 4) las habilidades para inventar e innovar en nuevas situaciones; y 5) las destrezas profesionales relacionadas con la tecnología. Por otro lado, Magro et ál. (2014) plantean cinco subdimensiones de competencia digital orientadas al fortalecimiento de las habilidades necesarias para que las personas se desenvuelvan en ambientes virtuales de aprendizaje (tabla 1).

Tabla 1. Definición de las dimensiones de competencia digital

\begin{tabular}{|l|l|}
\hline \multicolumn{1}{|c|}{ Subdimensiones } & \multicolumn{1}{c|}{ Definición } \\
\hline Comunicación digital & $\begin{array}{l}\text { Capacidad que presenta el estudiante virtual para comunicarse, } \\
\text { relacionarse y colaborar de forma eficiente con herramientas y en } \\
\text { entornos digitales para el cumplimiento de sus objetivos académicos. }\end{array}$ \\
\hline $\begin{array}{l}\text { Gestión de la } \\
\text { información }\end{array}$ & $\begin{array}{l}\text { Capacidad que tiene el estudiante virtual para buscar, obtener, } \\
\text { evaluar, organizar y compartir información en contextos académicos } \\
\text { digitales. }\end{array}$ \\
\hline Trabajo en red & $\begin{array}{l}\text { Capacidad que tiene el estudiante virtual para trabajar, colaborar y } \\
\text { cooperar en entornos académicos digitales. }\end{array}$ \\
\hline Aprendizaje continuo & $\begin{array}{l}\text { Capacidad que tiene el estudiante virtual para gestionar el aprendizaje } \\
\text { de manera autónoma, conocer y utilizar recursos digitales, mantener } \\
\text { y participar de comunidades de aprendizaje. }\end{array}$ \\
\hline Conocimiento digital & $\begin{array}{l}\text { Capacidad que tiene el estudiante virtual para desenvolverse } \\
\text { académica y personalmente en entornos virtuales. }\end{array}$ \\
\hline
\end{tabular}

Fuente: Magro et ál. (2014).

En Colombia, para el Ministerio de Educación Nacional (2006) una competencia tecnológica se define como la "capacidad para seleccionar y utilizar de forma pertinente, responsable y eficiente una variedad de herramientas tecnológicas entendiendo los principios que las rigen, la forma de combinarlas y las licencias que las amparan" (p. 36).

Por otro lado, es importante mencionar que las competencias tecnológicas pueden enmarcarse conceptualmente en el constructo de la alfabetización digital (AD) que, para García (2017), implica que las personas adquieran y desarrollen aptitudes transferibles y utilizables a lo largo de toda la vida para la resolución de problemas. De igual forma, la $\mathrm{AD}$ busca que en el área educativa se desarrollen pedagogías activas centradas en el estudiante y enfocadas en los recursos y en la solución de problemas de la vida real; mientras que, en el ámbito sociolaboral, hace énfasis en el dominio del análisis, la gestión, recuperación y evaluación de información electrónica. Si bien los estudiantes de Psicología deben desarrollar competencias digitales enfocadas a la carrera, también deben desarrollar habilidades enfocadas a su desarrollo laboral; por ende, como formadores deben cumplir con esta funcionalidad.

La Unesco (2006) considera que la AD es necesaria para el logro de los objetivos del aprendizaje, puesto que vincula las nuevas tecnologías y posibilita la disminución de la brecha de cobertura en los sistemas educativos. Por lo tanto, el sistema de enseñanza a distancia puede ofrecer posibilidades importantes para que las personas se formen en distintas modalidades de educación a distancia.
Diferentes investigaciones apuntan a que, en pocos años, el perfil del estudiante universitario cambiará debido a la influencia de la sociedad del conocimiento, una sociedad altamente tecnológica que, entre sus características, según la Fundación Telefónica (2012), se destacará por una mayor inteligencia visual, preferencia por la hipertextualidad, inmediatez, flexibilidad, mayor capacidad de resolución de problemas, capacidad de socialización en red y por estar al día en cuanto a nuevos productos y servicios tecnológicos. Por ende, la universidad se verá obligada a ajustarse a ese nuevo perfil de alumno, con la trasformación de herramientas, de metodología y, hasta en algunos casos, la forma de entender los procesos de enseñar y aprender.

A través del uso de las plataformas Moodle y Blackboard Collaborate no solo se genera un espacio para subir contenidos e interactuar con los estudiantes virtualmente, sino que se direcciona un proceso de enseñanza-aprendizaje. Para Duarte (2003) un ambiente educativo es un escenario donde existen y desarrollan condiciones favorables de aprendizaje; un espacio físico o virtual donde los participantes desarrollan capacidades, competencias, habilidades y valores. Asimismo, el concepto de AVA es referente para las organizaciones, comunidades, actividades y prácticas que operan y tienen lugar en internet; se subraya su potencialidad por permitir una comunicación entre usuarios, similar a la que se realiza cara a cara (Coll y Monereo, 2008, citado en Valencia, Huertas y Baracaldo, 2014). 


\section{Percepción sobre el aprendizaje}

A pesar de la cantidad de literatura científica que muestra las bondades de la innovación digital en el campo de la enseñanza, es común encontrar que en el contexto de la educación superior se privilegian acciones basadas en el resultado, en parte por el carácter subjetivo que implica la inclusión de aspectos más flexibles como la autoevaluación, la posibilidad de que los estudiantes puedan elegir los contenidos a los que acceden o considerar las percepciones de los estudiantes frente al aprendizaje en los cursos (Cruz y Quiñones, 2012). La evaluación sumativa, por ejemplo, no ofrece per se devoluciones que inviten a la reflexión y tampoco oportunidades de autorregulación de los procesos de aprendizaje por parte de los estudiantes. Además, en el caso de la educación virtual, la automatización del proceso educativo, aunado a la administración curricular, ha hecho que el aspecto sumativo se fortalezca y se estandarice, imposibilitando, en muchos casos, el acceso a esos elementos más subjetivos que escapan de la mirada tradicional de la educación (Dorrego, 2016).

En relación con este último aspecto, existe evidencia empírica que vincula de forma positiva (en términos estadísticos) los resultados de las evaluaciones con la percepción del aprendizaje por parte de los estudiantes (O'Connell y Dickinson, 1993; Ryan y Harrison, 1995; Cashin y Downey, 1995). Además, algunos estudios manifiestan que los estudiantes con percepciones favorables hacia los temas y la metodología de los cursos pueden aprovechar en mayor medida los diferentes contenidos (Bonwell y Eison, 1991; Centra y Gaubatz, 2000; Pegalajar Palomino, 2016).

Una de las aproximaciones conceptuales más aceptadas del término percepción es la planteada por Barthey (1982; citado por Arias, 2006), quien lo define como "un conocimiento inmediato o intuitivo, o juicio; un discernimiento análogo a la percepción sensorial con respecto a su inmediatez y al sentimiento de certidumbre que lo acompaña, frecuentemente implica una observación agradable o una discriminación sutil" (p. 10). La percepción, entonces, es un insumo elemental para el establecimiento de juicios. A este punto, resulta pertinente mencionar el significado del término percepción de aprendizaje que se asume en el presente trabajo, para ello se toma como referente la aproximación de Castaño, Jenaro y Flores (2017), quienes lo definen como un conjunto de "valoraciones de aspectos relativos a los contenidos, metodología, profesorado y satisfacción de tal metodología” (p. 7). Si bien esta definición se da en una investigación desarrollada para conocer las percepciones de los estudiantes en un curso llevado a cabo en modalidad blended, funcionan para el propósito del presente porque son elementos que pueden generalizarse a la estructura de la modalidad a distancia virtual.

\section{Metodología}

Esta investigación es de tipo cuantitativo descriptivo, con diseño no experimental, de corte transversal, el cual, según Hernández, Fernández y Baptista (2016), recoge datos en un único momento para probar hipótesis con base en la medición numérica y el análisis estadístico. La población seleccionada para esta investigación es de 300 estudiantes de primer semestre de Psicología en modalidad virtual, entre los 16 y los 54 años, hombres y mujeres, con diversos niveles de escolaridad (mínimo de bachillerato), pertenecientes a los estratos socioeconómicos 2 a 4, de 15 departamentos de Colombia más Bogotá. El muestreo fue de carácter no probabilístico intencional de 131 estudiantes de primer semestre de Psicología, modalidad virtual, participantes del curso de alfabetización digital.

\section{Instrumento}

Cuestionario de percepciones frente al aprendizaje de
competencias digitales

Este cuestionario fue diseñado por el equipo de investigación y consta de 25 afirmaciones que indagan la percepción de los estudiantes sobre el aprendizaje de competencias digitales adquiridas en el programa de alfabetización digital mediado por un AVA. Este instrumento tiene un escalamiento tipo Likert con cinco niveles de respuesta, a saber: totalmente de acuerdo, de acuerdo, ni de acuerdo ni en desacuerdo, en desacuerdo y totalmente en desacuerdo.

La validación de contenido se realizó con base en la experiencia de profesionales e investigadores en el campo de la investigación educativa y la educación virtual, proceso que arrojó un coeficiente de validez de contenido (CVC) superior a 0.80 para cada uno de los ítems. Según Hernández\#Nieto (2002), citado por Pedrosa, Suárez y García (2013), esto se considera aceptable para cada reactivo. Posterior a esto se ejecutaron las correcciones sugeridas por cada uno de los jurados. En relación con el análisis de confiabilidad, se registraron resultados diferentes para cada dimensión: comunicación digital $(\#=0.96)$; gestión de la información (\# = 0.92) y trabajo en red (\# = 0.93); aprendizaje continuo $(\#=0.94)$ y conocimiento digital $(\#=0.95)$. Estos resultados aseguran la consistencia interna del instrumento.

La estructura factorial del instrumento está basada en cinco dimensiones: comunicación digital, gestión de la información, trabajo en red, aprendizaje continuo y conocimiento digital. La distribución de los ítems en las dimensiones del test se presenta en la tabla 2. 
Tabla 2 Distribución de ítems por factor

\begin{tabular}{|l|l|}
\multicolumn{1}{|c|}{ Factor } & \multicolumn{1}{c|}{ Ittems } \\
\hline Factor 1. Conocimiento digital & $8,7,6,9,210$ \\
\hline Factor 2. Gestión de la información & $17,16,22,25,19,18,12$ \\
\hline Factor 3. Comunicación digital & $14,11,13,15,20$ \\
\hline Factor 4. Trabajo en red & $4,1,3$ \\
\hline Factor 5. Aprendizaje continuo & $5,23,24,21$ \\
\hline
\end{tabular}

Fuente: elaboración propia.

La anterior distribución surgió de procedimientos estadísticos que permitieron establecer la estructura dimensional del instrumento de medición, la cual fue determinada por el método de componentes principales. Para ello se utilizó la rotación Varimax con normalización Kaiser. Los valores de Kaiser Meyer Olkin $(\mathrm{KMO}=0.863)$ y el test de esfericidad de Barlett $(\mathrm{p}<0.05)$ fueron los esperados para realizar el análisis factorial; por lo tanto, las saturaciones factoriales del cuestionario son las siguientes: 
Tabla 3. Ítems y saturaciones factoriales del cuestionario

'percepciones frente al aprendizaje de competencias digitales'

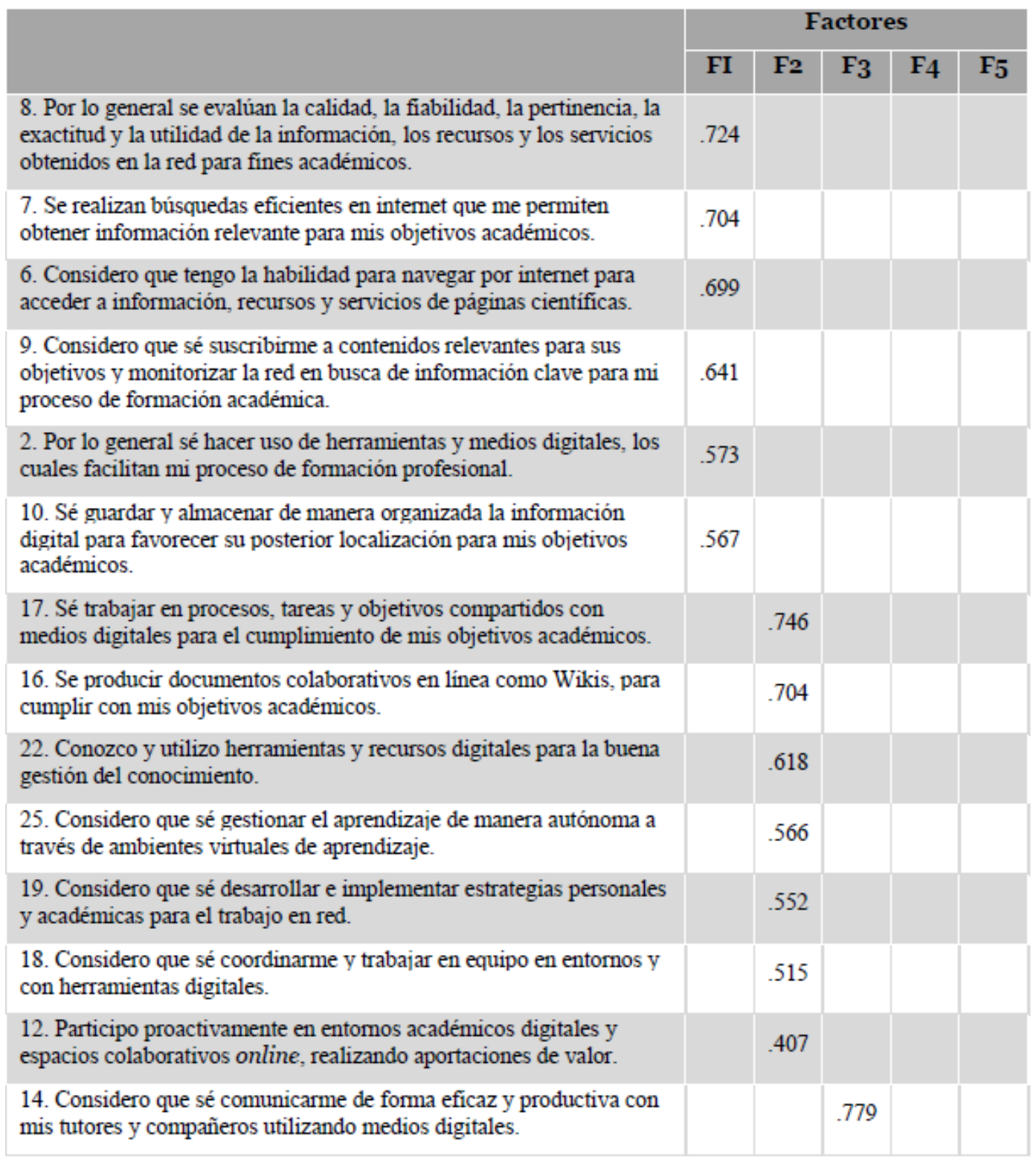

Fuente: elaboración propia. 
Tabla 3. Ítems y saturaciones factoriales del cuestionario 'percepciones frente al aprendizaje de competencias digitales'

\begin{tabular}{|c|c|c|c|c|c|}
\hline & \multicolumn{5}{|c|}{ Factores } \\
\hline & FI & F2 & $F_{3}$ & $F_{4}$ & $F_{5}$ \\
\hline $\begin{array}{l}\text { 11. Sé comunicarme de forma eficiente de manera asincrona con mis } \\
\text { tutores y compañeros de estudio. }\end{array}$ & & & .689 & & \\
\hline $\begin{array}{l}\text { 13. Sé comunicarme de forma eficiente de manera sincrona online, } \\
\text { con herramientas como Hangouts o Blackboard. }\end{array}$ & & & .673 & & \\
\hline $\begin{array}{l}\text { 15. Tengo la habilidad para establecer relaciones y contactos } \\
\text { académicos a través de medios digitales. }\end{array}$ & & & .523 & & \\
\hline $\begin{array}{l}\text { 20. Considero que el trabajo en equipo a través de la red promueve la } \\
\text { creatividad y la innovación, generando mejores productos } \\
\text { académicos. }\end{array}$ & & & .483 & & \\
\hline $\begin{array}{l}\text { 4. Hago un uso adecuado de herramientas digitales para el } \\
\text { pensamiento reflexivo, la creatividad y la innovación. }\end{array}$ & & & & .752 & \\
\hline $\begin{array}{l}\text { 1. Considero que utilizo de forma eficiente los recursos y } \\
\text { herramientas digitales para mi proceso de formación como estudiante } \\
\text { virtual. }\end{array}$ & & & & .705 & \\
\hline $\begin{array}{l}\text { 3. Considero que intervengo de forma responsable, segura y ética en } \\
\text { entornos digitales institucionales o propios. }\end{array}$ & & & & .548 & \\
\hline $\begin{array}{l}\text { 5. Considero que las herramientas digitales, como Mindomo, } \\
\text { permiten una mayor gestión del tiempo, facilitan el trabajo, aceleran } \\
\text { la producción de conocimiento e incrementan la creatividad. }\end{array}$ & & & & & .636 \\
\hline $\begin{array}{l}\text { 23. Participo en actividades de formación gratuita, pero formal en } \\
\text { linea, como MOOCS, para complementar mi formación académica. }\end{array}$ & & & & & .583 \\
\hline $\begin{array}{l}\text { 24. Contribuyo en el aprendizaje entre iguales en entomos virtuales } \\
\text { de aprendizaje. }\end{array}$ & & & & & .518 \\
\hline $\begin{array}{l}\text { 21. Considero que como estudiante virtual sé gestionar mi propia } \\
\text { capacitación digital. }\end{array}$ & & & & & .492 \\
\hline
\end{tabular}

Fuente: elaboración propia.

\section{Resultados y análisis}

A continuación se presentan los resultados y los análisis descriptivos relacionados con la percepción de aprendizaje de los estudiantes en el curso de alfabetización digital. Primero, se muestran los resultados descriptivos básicos correspondientes a los factores de la escala, para después exponer los análisis de los resultados con base en dos variables de agrupación: género y nivel de escolaridad. Vale la pena resaltar que para las variables cuantitativas se efectuó la prueba de Kolmogorov\#Smirnof, la cual arrojó resultados que confirman la normalidad de las distribuciones $(\mathrm{p}<0.05)$.
En relación con el análisis descriptivo por factor, debe resaltarse que, en función de los valores máximos y mínimos en cada uno, la mayoría de las respuestas estuvieron cercanas al valor máximo de cada factor; evidencia de esto es el valor de las medidas de tendencia central y el coeficiente de asimetría. Por otro lado, la curtosis y la desviación estándar evidencian agrupación de los datos, lo que implica una baja dispersión de estos; por lo tanto, es posible afirmar que la percepción de los estudiantes frente al aprendizaje en el curso de alfabetización académica es homogénea y alta. La información está resumida en la tabla 4. 
Tabla 4. Estadísticos descriptivos

\begin{tabular}{|c|c|c|c|c|c|}
\hline & $\begin{array}{c}\text { Conocimiento } \\
\text { digital }\end{array}$ & $\begin{array}{l}\text { Gestión de } \\
\text { la } \\
\text { información }\end{array}$ & $\begin{array}{l}\text { Comunicación } \\
\text { digital }\end{array}$ & $\begin{array}{l}\text { Trabajo } \\
\text { en red }\end{array}$ & $\begin{array}{l}\text { Aprendizaje } \\
\text { continuo }\end{array}$ \\
\hline Media & $27 \cdot 77$ & $33 \cdot 31$ & 23.70 & $14 \cdot 7^{2}$ & 16.90 \\
\hline Mediana & 30.00 & 35.00 & 25.00 & 15.00 & 18.00 \\
\hline Moda & 30 & 35 & 25 & 15 & 18 \\
\hline $\begin{array}{l}\text { Desviación } \\
\text { estándar }\end{array}$ & 3.292 & $3 \cdot 335$ & 2.259 & 1.047 & 2.449 \\
\hline Varianza & 10.839 & 11.124 & 5.103 & 1.097 & 5.998 \\
\hline Asimetría & -1.686 & -2.637 & -2.664 & $-5 \cdot 533$ & -.847 \\
\hline $\begin{array}{l}\text { Error } \\
\text { estándar de } \\
\text { asimetría }\end{array}$ & .212 & .212 & .212 & .212 & .212 \\
\hline Curtosis & 2.147 & 6.878 & 9.145 & $3^{8.770}$ & .489 \\
\hline $\begin{array}{l}\text { Error } \\
\text { estándar de } \\
\text { curtosis }\end{array}$ & .420 & .420 & .420 & .420 & .420 \\
\hline Mínimo & 16 & 19 & 11 & 6 & 9 \\
\hline Máximo & 30 & 35 & 25 & 15 & 20 \\
\hline
\end{tabular}

Fuente: elaboración propia.

La información de la tabla 5 muestra que las respuestas por género fueron homogéneas en las cinco dimensiones del test, a excepción del factor trabajo en red, cuyos valores de desviación estándar muestran mayor dispersión en las respuestas de las mujeres. Esta diferencia podría ser explicada por una mayor cantidad de mujeres participantes (104 mujeres y 27 hombres). Por otro lado, en relación con el primer factor (conocimiento digital) se evidencian promedios diferentes; no obstante, al realizar el estadístico de comparación para grupos independientes, los resultados muestran que las diferencias entre ambos géneros no son significativas $(\mathrm{t}=0.183 ; \mathrm{p}>0.05)$. Un supuesto preliminar asociado al análisis de esta diferencia apunta a que, si bien las mujeres participantes tienen un mayor nivel de escolaridad en el grado de técnico y tecnólogo que los hombres, no es posible afirmar que el factor en cuestión se vea afectado por el logro de este nivel educativo. La información detallada sobre la escolaridad por género se presenta en la tabla 6 . 
Tabla 5. Media y desviación estándar de las puntuaciones en cada factor por género

\begin{tabular}{|l|l|r|r|}
\hline \multicolumn{1}{|c|}{ Factor } & & Media & Desviación estándar \\
\hline Conocimiento digital & Hombres & 28.37 & 2.306 \\
\hline \multirow{3}{*}{ Gestión de la información } & Mujeres & 27.62 & 3.496 \\
& Hombres & $33 \cdot 33$ & 2.496 \\
\hline \multirow{2}{*}{ Comunicación digital } & Mujeres & 33.31 & 3.531 \\
\hline \multirow{2}{*}{ Trabajo en red } & Hombres & 23.67 & 2.094 \\
\hline \multirow{2}{*}{ Aprendizaje continuo } & Mujeres & 23.71 & 2.309 \\
& Hombres & 14.85 & 0.534 \\
\hline & Mujeres & 14.68 & 1.143 \\
\hline & Hombres & 16.59 & 2.406 \\
\hline & Mujeres & 16.98 & 2.465 \\
\hline
\end{tabular}

Fuente: elaboración propia.

Tabla 6. Nivel de escolaridad por género

\begin{tabular}{|l|r|r|r|r|}
\hline & \multicolumn{2}{|c|}{ Mujeres } & \multicolumn{2}{|c|}{ Hombres } \\
\hline Bachilleres & \multicolumn{1}{|c|}{$\boldsymbol{f}$} & $\boldsymbol{H}$ & $\boldsymbol{f}$ & \multicolumn{1}{c|}{$\boldsymbol{H}$} \\
\hline TyT & $3^{1}$ & $29.8 \%$ & $\mathbf{1 1}$ & $40.7 \%$ \\
\hline Pregrado & 62 & $59.6 \%$ & $\mathbf{1 4}$ & $51.9 \%$ \\
\hline Posgrado & 9 & $\mathbf{8 . 7} \%$ & 2 & $7.4 \%$ \\
\hline Total & 2 & $\mathbf{1 . 9} \%$ & 0 & $0.0 \%$ \\
\hline
\end{tabular}

Fuente: elaboración propia.

Otro análisis propuesto fue comparar cada uno de los factores con el nivel de escolaridad de los participantes: bachillerato (32.06 $\%)$, técnico o tecnólogo (58.01\%), pregrado culminado (8.3\%) y especialización culminada $(1.5 \%)$. Los resultados del ANOVA (tabla 7) no muestran diferencias significativas asociadas con el nivel de escolaridad en ninguna dimensión. No obstante, los gráficos de promedios evidencian con mayor nivel de detalle las diferencias relacionadas con la percepción del aprendizaje en cada uno de los factores explorados (figura 1). 
Tabla 7. ANOVA por nivel de escolaridad

\begin{tabular}{|c|c|c|c|c|}
\hline & & Media cuadrática & $\mathbf{F}$ & Sig. \\
\hline \multirow{2}{*}{ Conocimiento digital } & Entre grupos & 6.592 & .603 & .614 \\
\hline & Dentro de grupos & 10.940 & & \\
\hline \multirow{2}{*}{ Gestión de la información } & Entre grupos & 20.043 & & .144 \\
\hline & Dentro de grupos & 10.914 & & \\
\hline \multirow{2}{*}{ Comunicación digital } & Entre grupos & $7 \cdot 403$ & 1.466 & .227 \\
\hline & Dentro de grupos & 5.049 & & \\
\hline \multirow{2}{*}{ Trabajo en red } & Entre grupos & $1.63^{8}$ & 1.512 & .215 \\
\hline & Dentro de grupos & 1.084 & & \\
\hline \multirow{2}{*}{ Aprendizaje continuo } & Entre grupos & 4.649 & $\cdot 771$ & $\cdot 512$ \\
\hline & Dentro de grupos & 6.030 & & \\
\hline
\end{tabular}

Fuente: elaboración propia. 
Figura 1. Promedio por nivel de escolaridad para los cinco
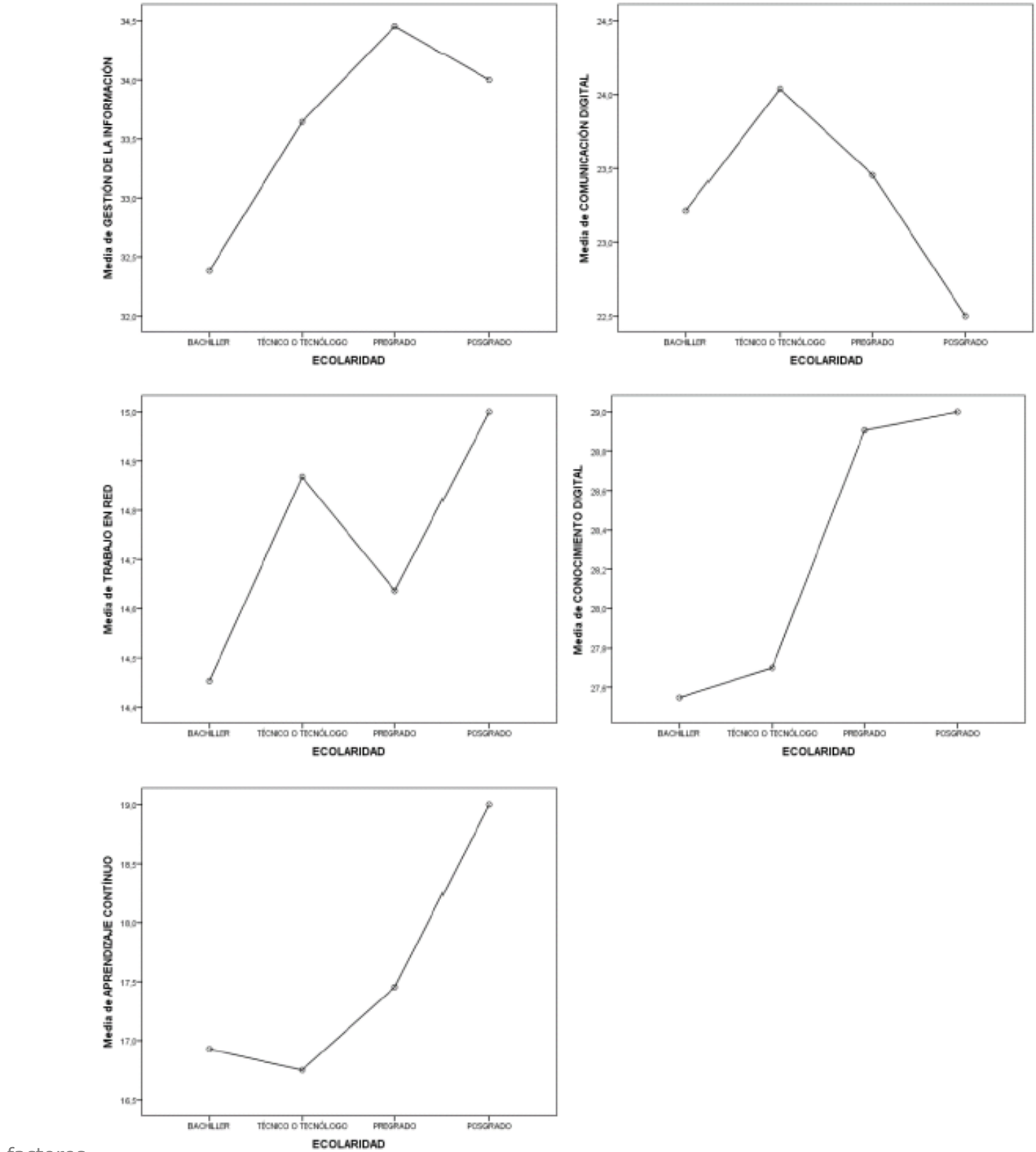

Fuente: elaboración propia.

La información de la figura 1 muestra que las personas con mayor nivel de escolaridad tienden a presentar una percepción mayor frente al aprendizaje de conocimientos digitales. Estos datos confirman lo expuesto en el aparatado donde se analizaron las diferencias entre género y escolaridad. En los niveles de bachillerato y TyT no se presentan diferencias significativas; sin embargo, las personas con pregrado y posgrado terminados evidencian percepciones más altas. Ahora, si bien es cierto que no existen diferencias significativas entre los grupos, es posible analizar que en los niveles de pregrado y posgrado se perciben mayores conocimientos digitales.

Una descripción detallada de este factor, en términos de los reactivos que lo estructuran, evidencia que los participantes con pregrado y posgrado terminado perciben mayores conocimientos frente a calidad, fiabilidad, pertinencia, exactitud y utilidad de la información; además, saben realizar búsquedas eficientes en internet para obtener información relevante que favorezca el 
logro de objetivos académicos y seleccionan fuentes válidas de información

Los valores del factor gestión de la información son similares a los de la dimensión anterior, en las dos se muestran mayores promedios en los niveles de pregrado y posgrado. Esta relación fue confirmada por medio de un análisis de correlación $(\mathrm{r}=0.6$; $\mathrm{p}<0.01$ ), que muestra que estos factores se asocian de forma significativa, es decir, a mayor percepción frente de aprendizaje de conocimientos digitales, mayor percepción de aprendizaje en gestión de la información. En síntesis, las personas con nivel de escolaridad de pregrado y posgrado perciben que el curso de alfabetización digital les permitió aprender a producir documentos colaborativos, gestionar mejor el aprendizaje de manera autónoma, a coordinarse y a trabajar en equipo en entornos digitales.

Contrario a los datos arrojados en los anteriores factores, los participantes que percibieron mayor aprendizaje en la dimensión de comunicación digital fueron los de nivel técnico y tecnólogo, información que podría implicar que los estudiantes aprovecharon en mayor medida los contenidos del curso implicados en el desarrollo de aprendizajes que favorecen el componente comunicativo. Una posible explicación al respecto es que estos contenidos fueron novedosos para ellos $\mathrm{y}$, por lo tanto, el promedio de las respuestas fue mayor en comparación con los demás factores. De forma particular, los estudiantes perciben aprendizajes relacionados con la comunicación eficaz y productiva con los tutores y los compañeros; además, aprendieron a utilizar herramientas como Hangouts o Blackboard para comunicarse de manera sincrónica.

En el factor de trabajo en red se identificaron resultados similares en todos los grupos, puesto que la diferencia cuantitativa entre promedios es de 0.6. No obstante, es pertinente resaltar que todos los participantes perciben que los aprendizajes en esta dimensión fueron altos si se considera que la puntuación máxima es 15. Los estudiantes perciben que aprendieron a utilizar de manera eficiente los recursos y herramientas digitales para mejorar el proceso de formación, también perciben que aprendieron a hacer uso adecuado de herramientas digitales para fortalecer el pensamiento reflexivo, la creatividad, la innovación y a intervenir de forma responsable, segura y ética en entornos digitales institucionales o propios. Además, esta dimensión está relacionada con el trabajo colaborativo, puesto que los actores del proceso formativo se comprometen a aprender en conjunto, por lo que el aprendizaje solo puede conseguirse si el trabajo en red se efectúa de forma mancomunada (Pérez, 2007).

Teniendo en cuenta que el factor de aprendizaje continuo se define de manera teórica como la capacidad que tienen los estudiantes virtuales para gestionar el aprendizaje de manera autónoma, conocer y utilizar recursos digitales, mantener y participar de comunidades de aprendizaje (Magro et ál., 2014). Podría inferirse que esta dimensión está relacionada con las necesidades de formación de los estudiantes y la posibilidad de cualificar la actividad profesional. Los resultados muestran una diferencia importante en el nivel de posgrado, lo que está asociado con los hallazgos de Chálela, Valencia y Arango (2007), donde algunos de los factores motivacionales de los estudiantes de formación avanzada para continuar aprendiendo tienen que ver con la valoración de la calidad académica de las instituciones; además, valoran las actividades académicas de apoyo como un elemento sustancial para el fortalecimiento de los aprendizajes.

Lo anterior queda evidenciado por la respuesta favorable en los reactivos que estructuran el factor; en otras palabras, los estudiantes con formación posgradual que cursaron el módulo de alfabetización académica perciben que las actividades de formación gratuita, pero formal en línea, como MOOCS, son importantes para complementar la formación académica.

\section{Conclusiones}

A modo de conclusión se resalta que las dimensiones de alfabetización digital seleccionadas permitieron desarrollar un instrumento con propiedades psicométricas aceptables para la medición de la percepción del aprendizaje; no obstante, es necesario ahondar en la estructura dimensional del instrumento con análisis que confirmen los factores establecidos. Pese a esto, se considera que este fue un insumo adecuado y pertinente para representar el constructo teórico y para el logro del propósito del presente estudio.

En relación con las percepciones de los estudiantes frente al aprendizaje en el curso de Alfabetización digital, no se evidencian diferencias estadísticamente significativas que apunten a que existe mayor o menor percepción de aprendizaje de los estudiantes en algún factor, por lo que se decidió realizar un análisis más detallado a partir de estadísticos de comparación entre variables categóricas como el género y el nivel de escolaridad. Los resultados muestran que existe una mayor percepción de aprendizaje por parte de los estudiantes que cuentan con nivel de posgrado y pregrado culminados, a excepción del factor de trabajo en red, que obtuvo mayores puntuaciones en los participantes que tienen estudios técnicos o tecnológicos.

En relación con el género, es posible evidenciar que no existen diferencias significativas en ninguno de los factores. Ahora, este resultado no pretende ser definitivo, esta información podría ser útil para el desarrollo de una fase posterior que profundice en otras variables demográficas como el número de hijos, tipo de familia, años de escolaridad u otras variables que se asocien al género. En ese sentido, es posible identificar si los roles sociales asignados a cada género se asocian en menor o mayor medida con las percepciones de aprendizaje en ambientes digitales.

Una de las conclusiones más importantes es que algunos hallazgos de este estudio deben ser profundizados mediante análisis cualitativos que aumenten la comprensión frente a las percepciones de aprendizaje de los estudiantes. Es decir, la pregunta por cuestiones asociadas con las experiencias e intenciones de formación y las condiciones de posibilidad de las personas que deciden adelantar su formación en modalidad virtual es obligada si se pretende hacer un análisis de carácter más holístico. Por lo tanto, este estudio de corte cuantitativo puede ser un insumo que aporte a la construcción de conocimiento, a propósito de la percepción sobre el aprendizaje en ambientes virtuales de formación; además, el cuestionario Percepciones frente al aprendizaje de competencias digitales podría ser utilizado por otros equipos de investigación como un elemento central o complementario en futuros estudios.

\section{Referencias}

Ardila, J., Ruiz, E. y Castro, I. (2015). Estudio comparativo de sistemas de gestión del aprendizaje: Moodle, ATutor, Claroline, Chamilo y Universidad de Boyacá . Revista Academia y Virtualidad, .(1), 54\#65. https://doi.org/10.18359/ravi.445

Arias, C. (2006). Enfoques teóricos sobre la percepción que tienen las personas. Horizontes pedagógicos, .(1), 9-22. https://horiz ontespedagogicos.ibero.edu.co/article/view/08101 
Bonwell, C. C. y Eison, J. A. (1991). Active learning: Creating excitement in the classroom. School of Education and Human Development, George Washington University.

Cashin, W. E. y Downey, R. G. (1995). Disciplinary differences in what is taught and in students' perceptions of what they learn and of how they are taught. En M. Theall y J. Franklin (eds.), New Directions for Teaching and Learning (p. 64). Jossey\#Bass.

Castaño, R., Jenaro, C. y Flores, N. (2017). Percepciones de estudiantes del Grado de Maestro sobre el proceso y resultados de la enseñanza semipresencial\#Blended Learning. Revista de Educación a Distancia , (52), 2.

Castellanos, O., Jiménez, C. y Domínguez, K. (2009). Competencias tecnológicas: bases conceptuales para el desarrollo tecnológico en Colombia. Revista Ingeniería e Investigación. 29 (1), 133\#139. http://www.scielo.org.co/pdf/ie i/v29n1/v29n1a17.pdf

Centra, J. A. y Gaubatz, N. B. (2000). Student perceptions of learning and instructional effectiveness in college courses. Research Rep, (9).

Chálela, S., Valencia, A. y Arango, D. (2017). Motivaciones de los estudiantes universitarios para continuar con su formación académica en programas de posgrado. Revista Lasallista de investigación. 14 (2), 160\#170.

Cruz, F. y Quiñones, A. (2012). Importancia de la evaluación y autoevaluación en el rendimiento académico. Zona Próxima , (16), 96\#104. https://www.redalyc.org/articulo.oa?id=853/85 323935009

Dorrego, E. (2016). Educación a distancia y evaluación del aprendizaje. Revista de Educación a Distancia (RED), 50, htt ps://revistas.um.es/red/article/view/271241

Duarte, J. (2003). Ambientes de aprendizaje: una aproximación conceptual. Estudios Pedagógicos. 29, 97\#113. http://www.re dalyc.org/pdf/1735/173514130007.pdf

Fundación Telefónica. (2012). Alfabetización digital y competencias informacionales . Fundación Telefónica.

García, S. (2017). Alfabetización Digital. Razón y Palabra. 21 (98). https://www.redalyc.org/articulo.oa?id=1995/199553113 006

Hernández. S, Fernández. C. y Baptista. P. (2016). Metodología de la investigación. McGraw Hill. https://unesdoc.unesco.org/ ark:/48223/pf0000147000

Magro, C., Salvatella, J., Álvarez, M., Herrero, O. Paredes, A. y Vélez, G. (2014). Cultura digital y transformación de las organizaciones: ocho competencias digitales para el éxito profesional . RocaSalvatella https://rocasalvatella.com/app/upl oads/2018/11/maqueta_competencias_espanol.pdf

Ministerio de Educación Nacional. (2006). Estándares básicos de competencias en tecnología e informática. Ser competente en tecnología ;Una necesidad para el desarrollo! Lo que necesitamos saber y saber hacer. http://www.colegionaciones unidasied.com/pdf/tecno.pdf

Miranda, G. (2004). De los ambientes virtuales de aprendizaje a las comunidades de aprendizaje en línea. Revista Digital Universitaria, .(10), 1\#15. http://www.revista.unam.mx/vol.5/n um10/art62/nov_art62.pdf

O'Connell, D. Q. y Dickinson, D. J. (1993). Student Ratings of Instruction as a Function of Testing Conditions and Perceptions of Amount Learned. Journal of Research and Development in Education. 27 (1), 18-23.
Palomo, M., (2001). Las ingenierías y la administración de la tecnología. Ingenierías, .(12), 9-14. https://es.calameo.com/ey a-academy/read/003506270a80938aa88a1

Pedrosa, I., Suárez, J. y García, E. (2013). Evidencias sobre la validez de contenido: avances teóricos y métodos para su estimación. Acción Psicológica. 10 (2), 3\#18. http://scielo.isci ii.es/pdf/acp/v10n2/02monografico2.pdf

Pegalajar, M. (2016). Percepciones del alumnado de posgrado acerca de la metodología docente y evaluativa. profesorado. Revista de Currículum y Formación de Profesorado. 20 (2), 98\#119. https://revistaseug.ugr.es/index.php/profesorado/articl e/download/10410/8508

Pérez, M. M. (2007). El trabajo colaborativo en el aula universitaria. Laurus. 13 (23), 263\#278. https://www.redalyc.o rg/pdf/761/76102314.pdf

Poriet, Y. y Martínez, O. (2014). Competencias tecnológicas en la gerencia universitaria. Orbis. Revista Científica Ciencias Humanas, . (27), 47\#67. http://www.redalyc.org/pdf/709/70930 407003.pdf

Ryan, J. y Harrison, P. (1995). The relationship between individual instructional characteristics and the overall assessment of teaching effectiveness across different instructional contexts. Research in Higher Education. 36 (5), 577\#594.

Unesco (2006). La alfabetización, un factor vital. Informe de seguimiento de la Educación para todos en el mundo. Unesdoc. https://unesdoc.unesco.org/ark:/48223/pf0000147000

Valencia, N., Huertas, A. y Baracaldo, B. (2014). Los ambientes virtuales de aprendizaje: una revisión de publicaciones entre 2003 y 2013, desde la perspectiva de la pedagogía basada en la evidencia. Revista Colombiana de Educación. 66 . http://www .scielo.org.co/pdf/rcde/n66/n66a04.pdf

\section{Bibliografía consultada}

Arrieta, A. y Montes, D. (2011). Alfabetización digital: uso de las TIC más allá de una formación instrumental y una buena infraestructura. Revista Colombiana De Ciencia Animal - RECIA, 3(1), 180-197. https://doi.org/10.24188/recia.v3.n1.2 011.360

Castellanos, A., Sánchez, C. y Calderero, J. (2017). Nuevos modelos tecnopedagógicos. Competencia digital de los alumnos universitarios. Revista electrónica de investigación educativa, 19(1), 1\#9. https://dx.doi.org/10.24320/redie.2017.19.1.1148

Chou, R., Valdés, A. y Sánchez, S. (2017, marzo 20). Programa de formación de competencias digitales en docentes universitarios. Revista Universidad y Sociedad, 9(1), 81\#86. http://scielo.sld.cu/scielo.php?script=sci_arttext\&pid=S 221836202017000100011\&lng=es\&tlng=es

Moodle (2020). Plataforma de aprendizaje todo en uno. https://do cs.moodle.org/all/es/Acerca_de_Moodle

Olave, G., Rojas, L. y Cisneros, M. (2013). Deserción universitaria y alfabetización académica. Educación y Educadores, 16(3), 455\#471. https://educacionyeducadores.unisabana.edu.co/inde x.php/eye/article/view/2726/3352

Quintela, G. (2013). Deserción universitaria, una aproximación sociológica al proceso de toma de decisiones de los estudiantes. Sociedad Hoy, (24). https://www.redalyc.org/articulo.oa?id=90 2/90231580008 
Notas

* Texto presentado en el IV Foro Internacional de Investigación en Ambientes Virtuales de Aprendizaje

(ㄷ) (1) (3) (3) 